\title{
STRESS STATE IN FRONT OF A CRACK PERPENDICULAR TO BIMATERIAL INTERFACE
}

\author{
T. C. WANG $\dagger$ \\ LNM, Institute of Mechanics, Chinese Academy of Sciences, Beijing, 100080, People's Republic of \\ China \\ P. STÅHLE \\ Division of Solid Mechanics, Luleå University of Technology, S-97187 Luleå, Sweden
}

\begin{abstract}
Using a dislocation simulation approach, the basic equation for a crack perpendicular to a bimaterial interface is formulated in this paper. A novel expansion method is proposed for solving the problem. The complete solution for the problem, including the $T$ stress ahead of the crack tip and the stress intensity factors are presented. The stress field characteristics are analyzed in detail. It is found that ahead of the crack tip and near the interface the normal stress, perpendicular to the crack plane, $\sigma_{x}$, is characterized by the $\mathrm{K}$ fields and the normal stress $\sigma_{y}$ is dominated by the $\mathrm{K}$ field plus $T$ stress in the region of $0<r / b<0.4$ for $b / a_{0} \leq 0.1$, where $b$ is the distance from the crack tip to the interface. (C) 1998 Elsevier Science Ltd. All rights reserved
\end{abstract}

Keywords-bimaterials interface, dislocation simulation, dominated zone, perpendicular crack, stress intensity factor, $T$ stress.

\section{NOMENCLATURE}

\begin{tabular}{|c|c|}
\hline$x, y$ & rectangular coordinate, $x$ axis is along the interface \\
\hline$a$ & distance from the crack tip $A$ to the interface \\
\hline$b$ & distance from the crack tip $B$ to the interface \\
\hline$c$ & distance from the center of the crack to the interface, $c=(a+b) / 2$ \\
\hline$a_{0}$ & a half of the crack length, $a_{0}=(a-b) / 2$ \\
\hline$s_{x}, s_{y}, \tau_{x y}$ & stress components \\
\hline $\begin{array}{l}u_{x}, u_{y} \\
\Phi(z), \Omega(z)\end{array}$ & $\begin{array}{l}\text { displacements in the }(x, y) \text { direction } \\
\text { complex potentials }\end{array}$ \\
\hline$\mu$ & shear modulus \\
\hline$\nu$ & poisson's ratio \\
\hline$\kappa=3-4 v$ & for plane strain \\
\hline$\alpha, \beta$ & Dunders's parameters \\
\hline$K_{\mathrm{I}}$ & stress intensity factor \\
\hline$T$ & $T$ stress, characterizing the second term of the Williams series. \\
\hline
\end{tabular}

\section{INTRODUCTION}

THE ADVANCED materials, such as fiber or particle reinforced composites, metal-ceramics interfaces, laminated ceramics, adhesive joints, etc. have many important applications in the combustion engine component, aerospace bearing, cutting tools, hip joints, components for printing industry, transmission elements etc. Their behavior on fatigue, corrosion or wear can be significantly improved by surface coating. Thin films of metals, ceramics and polymers deposited on tools, machinery or electronic devices can endow the structures with new thermal, mechanical, chemical and tribological properties.

Interfaces failures are a common features in the advanced materials and thin films. The design process of these components requires a better understanding of the failure mechanisms of these components. An important task is devoted to detailed investigations on the fracture characteristics of flaws along or perpendicular to the interface. 


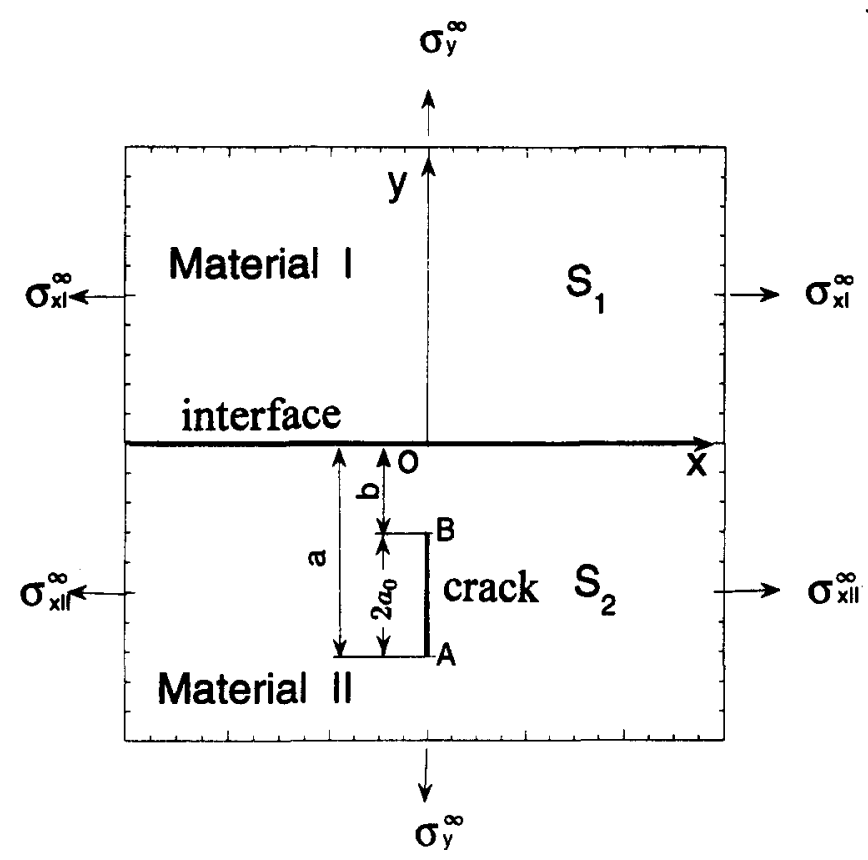

Fig. 1. A finite crack perpendicular to a bimaterial interface.

A crack perpendicular to a bimaterial interface has attracted many investigations. Zak and Williams [1] used the eigenfunction expansion method to analyze the stress singularity ahead of a crack tip, which is perpendicular to and terminating at the interface. Their analysis revealed that the stress singularity is of the order $r^{-\lambda}$, where $\lambda$ is a real eigenvalue and dependent on the elastic moduli of the bimaterial. Cook and Erdogen[2] used the Mellin transform method to derive the governing equation of a finite crack perpendicular to the interface and obtained the stress intensity factors. Erdogan and Biricikoglu [3] solved the problem of two bounded half planes with a crack going through the interface. Bogy [4] investigated the stress singularity of an infinite crack terminated at the interface with an arbitrary angle. Wang and Chen [5] used photoelasticity to determine the stress distribution and the stress intensity factors of a crack perpendicular to the interface. They found that the far-field has significant effect on the stress distribution and the stress intensity factors. Lin and Mar [6] presented a finite element analysis of the stress intensity factors for cracks perpendicular to a bimaterial interface. Ahmad [7] used the finite element method to analyze a crack normal to the interface in unidirectional fiber composites. Meguid et al. [8] proposed a novel finite element to analyze cracks perpendicular to bimaterial in finite elastic body. Chen [9] used the body force method to determine the stress intensity factors for a crack normal to and terminated at a bimaterial interface. His results are in good agreement with the results by Meguid et al. [8]. Ståhle et al.[10,11] investigated a crack growing towards to a bimaterial interface. They carried out an experiment work and a finite element simulation. Their results showed that the crack can be deflected and follows a smooth curved path.

Using dislocation simulation approach, the basic equation for a crack perpendicular to a bimaterial is formulated in this paper. A novel expansion method is proposed for solving the basic equation. The dislocation density is expressed as a series of the first Chebyshev polynomial with a set of unknown coefficients. Then the complex potential and the stress fields can be expanded as a power series of a new complex variable, which is a simple rational function of the complex variable $z$. Combined with the boundary collocation method, the governing equation is solved and the unknown coefficients are obtained. The complete solution for the problem and the stress intensity factors are presented. 


\section{BASIC FORMULAE}

Consider the plane elastic problem as shown in Fig. 1. A finite crack is perpendicular to a bimaterial interface. A Cartesian coordinate system oxy is attached on the interface. The $x$ axis is along the interface and the $y$ axis is normal to the interface and coincident with the crack elongation direction. Both materials are isotropic and homogeneous. The material $I$ is occupied the upper half plane $S_{1}$ and the materials II is occupied the lower half plane $S_{2}$.

\subsection{Complex potential}

Stress and displacement in an elastic solid can be represented by two Muskhelishivili's potentials:

$$
\begin{gathered}
\sigma_{x}+\sigma_{y}=4 \operatorname{Re}(\Phi(z)) \\
\sigma_{y}-i \tau_{x y}=\Phi(z)+\Omega(\bar{z})+(z-\bar{z}) \overline{\Phi^{\prime}(z)} \\
2 \mu\left(u_{x}+i u_{y}\right)=\kappa \phi(z)-\omega(\bar{z})-(z-\bar{z}) \overline{\Phi(z)} .
\end{gathered}
$$

The complex potentials for an edge dislocation at $z=s$ in an infinite elastic solid can be expressed as follows:

$$
\begin{gathered}
\Phi_{0}(z)=\frac{B}{z-s} \\
\Omega_{0}(z)=\frac{B}{z-\bar{s}}+\bar{B} \frac{s-\bar{s}}{(z-\bar{s})^{2}} \\
B=\frac{\mu}{\pi i(\kappa+1)}\left(b_{x}+i b_{y}\right),
\end{gathered}
$$

where $b_{x}$ and $b_{y}$ are the $x$ - and $y$-components of the dislocation, $\kappa=3-4 v$ for plane strain, $\nu$ is Poisson's ratio and $\mu$ is the shear modulus.

The interaction problem of an edge dislocation with a bimaterial interface was studied by Dundurs [12], Tamate [13] and Tamate and Kurihara [14] and Suo [15] among others. If the edge dislocation is embedded in material II, the complex potentials are (see Suo[15]):

$$
\begin{gathered}
\Phi(z)= \begin{cases}\left(1+\Lambda_{1}\right) \Phi_{0}(z), & z=S_{1}, \\
\Phi_{0}(z)+\Lambda_{2} \Omega_{0}(z), & z \in S_{2},\end{cases} \\
\Omega(z)= \begin{cases}\Phi_{0}(z)+\Lambda_{1} \Omega_{0}(z), & z \in S_{1} \\
\left(1+\Lambda_{2}\right) \Phi_{0}(z), & z=S_{2},\end{cases} \\
\Lambda_{1}=\frac{\alpha+\beta}{1-\beta} \\
\Lambda_{2}=\frac{\alpha-\beta}{1+\beta},
\end{gathered}
$$

where $\alpha$ and $\beta$ are two Dundur's parameters

$$
\begin{gathered}
\alpha=\frac{\left(\kappa_{2}+1\right) / \mu_{2}-\left(\kappa_{1}+1\right) / \mu_{1}}{\left(\kappa_{2}+1\right) / \mu_{2}+\left(\kappa_{1}+1\right) / \mu_{1}} \\
\beta=\frac{\left(\kappa_{2}-1\right) / \mu_{2}-\left(\kappa_{1}-1\right) / \mu_{1}}{\left(\kappa_{2}+1\right) / \mu_{2}+\left(\kappa_{1}+1\right) / \mu_{1}} .
\end{gathered}
$$

The crack can be considered as a continuous distribution of dislocations. Hence for our problem, we have: 


$$
\begin{gathered}
\Phi_{0}(z)=\frac{\mu_{2}}{\pi i\left(1+\kappa_{2}\right)} \int_{b}^{a} \frac{\left(b_{x}+i b_{y}\right)}{z+i t} \mathrm{~d} t \\
\Omega_{0}(z)=\frac{\mu_{2}}{\pi i\left(1+\kappa_{2}\right)} \int_{b}^{a} \frac{\left(b_{x}+i b_{y}\right)}{z-i t} \mathrm{~d} t+\frac{2 \mu_{2}}{\pi\left(1+\kappa_{2}\right)} \int_{b}^{a} \frac{t\left(b_{x}-i b_{y}\right)}{(z-i t)^{2}} \mathrm{~d} t,
\end{gathered}
$$

where $a$ and $b$ are the distances from the crack tips $A$ and $B$ to the interface, respectively. Introduce a new complex variable $z^{*}$ and a new function $I\left(z^{*}\right)$,

$$
\begin{gathered}
z^{*}=i z \\
I\left(z^{*}\right)=\frac{1}{\pi} \int_{b}^{a} \frac{b_{x}+i b_{y}}{z^{*}-t} \mathrm{~d} t .
\end{gathered}
$$

The function $I\left(z^{*}\right)$ is a holomophic function in the whole complex plane $z^{*}$ outside the cut $(b, a)$. Using the following variable transformations:

$$
z^{*}=\frac{a+b}{2}+\frac{a-b}{2} \zeta, t=\frac{a+b}{2}+\frac{a-b}{2} \xi,
$$

the function $I\left(z^{*}\right)$ can be represented as

$$
I\left(z^{*}\right)=\frac{1}{\pi} \int_{-1}^{1} \frac{b_{x}+i b_{y}}{\zeta-\xi} \mathrm{d} \xi .
$$

Assume the dislocation density can be expanded as a series of the first Chebyshev polynomial

$$
b_{x}+i b_{y}=\frac{1}{\sqrt{1-\xi^{2}}} \sum_{m=0}^{\infty} \alpha_{m} T_{m}(\xi)
$$

where $T_{m}(\xi)$ is the first Chebyshev polynomial

$$
T_{m}(\xi)=\cos m \theta, \xi=\cos \theta
$$

The components $b_{x}$ and $b_{y}$ of the dislocation density have the square-root singularity $r^{-1 / 2}$ at the crack tips. The first term of the right-hand side of the eq. (8) characterizes the desired singularity at the crack tips. In order to get a closed analytical solution of eq. (7), the series of the first Chebyshev polynomial is introduced in eq. (8).

The opening displacement on the crack surface can be expressed as:

$$
\delta_{x}+i \delta_{y}=-\int_{b}^{t}\left(b_{x}+i b_{y}\right) \mathrm{d} t=-\int_{-1}^{\xi} \frac{1}{\sqrt{1-\xi^{2}}} \sum_{m=0}^{\infty} \alpha_{m} T(\xi) \mathrm{d} \xi a_{0}=a_{0} \alpha_{0}(\pi-\theta)+a_{0} \sum_{m=1}^{\infty} \alpha_{m} \frac{\sin m \theta}{m},
$$

where $a_{0}$ is a half of the crack length, $c$ is the distance from the center of the crack to the interface as shown in Fig. 1. We have:

$$
\begin{gathered}
a_{0}=(a-b) / 2 \\
c=(a+b) / 2 \\
\xi=\cos \theta=(t-c) / a_{0} .
\end{gathered}
$$

Since the opening displacement at point A should be zero. It means that when $t=a, \xi=1$ and $\theta=0$, the opening displacement equals zero; this leads to $\alpha_{0}=0$.

Substituting eq. (8) into eq. (7) and with the aids of the following equation (see Gladwell[16]):

$$
\frac{1}{\pi} \int_{-1}^{1} \frac{T_{m}(x)}{\sqrt{1-x^{2}}(z-x)} \mathrm{d} x=\frac{1}{\sqrt{z^{2}-1}}\left[z-\sqrt{z^{2}-1}\right]^{m}
$$


we obtain

$$
I\left(z^{*}\right)=\frac{1}{\sqrt{\zeta^{2}-1}} \sum_{m=1}^{\infty} \alpha_{m}\left[\zeta-\zeta^{2}-1\right]^{m}, \zeta=\frac{\left(z^{*}-c\right)}{a_{0}}
$$

\subsection{Stress jump across inreference}

The displacements $u_{x}$ and $u_{y}$ should be continuous across the interface. Hence the strain $\varepsilon_{x}$ should be continuous across the interface. It follows:

$$
\left(\varepsilon_{x}\right)_{\mathrm{I}}=\left(\varepsilon_{x}\right)_{\mathrm{II}} \text {. }
$$

For the plane strain problem from this equation, we obtain directly:

$$
\left(\sigma_{x}\right)_{\mathrm{I}}=\frac{\mu_{1}\left(1-v_{2}\right)}{\mu_{2}\left(1-v_{1}\right)}\left(\sigma_{x}\right)_{\mathrm{II}}+\frac{\sigma_{y}}{1-v_{1}}\left(\nu_{1}-\frac{\mu_{1}}{\mu_{2}} \nu_{2}\right) .
$$

The above equation can be represented as:

$$
\left(\sigma_{x}\right)_{\mathrm{I}}=\frac{(1+\alpha)}{(1-\alpha)}\left(\sigma_{x}\right)_{\mathrm{II}}+\frac{2 \sigma_{y}}{(1-\alpha)}(2 \beta-\sigma)
$$

Of course, this equation is also suitable for plane stress problem. We should emphasis that the stress jumping eq. (13) is important for the insight understanding the interface problem.

At infinity, we have:

$$
\left(\sigma_{x}^{\infty}\right)_{\mathrm{I}}=\frac{(1+\alpha)}{(1-\alpha)}\left(\sigma_{x}^{\infty}\right)_{\mathrm{II}}+\frac{2 \sigma_{y}^{\infty}}{(1-\alpha)}(2 \beta-\alpha)
$$

\subsection{Superposition scheme}

On the crack surfaces, the traction-free condition should be satisfied. According to the superposition scheme, we need two solutions. The first solution is that for an infinite bimaterial without any crack subject to uniform remote loading. We only consider the symmetric problem. The skew-symmetric problem can be treated in a similar way.

The first solution for the symmetrical problem is:

$$
\begin{gathered}
\sigma_{x}=\left(\sigma_{x}^{\infty}\right)_{\mathrm{I}}, \sigma_{y}=\sigma_{y}^{\infty}, \tau_{x y}=0, \text { in material I } \\
\sigma_{x}=\left(\sigma_{x}^{\infty}\right)_{\mathrm{II}}, \sigma_{y}=\sigma_{y}^{\infty}, \tau_{x y}=0, \text { in material II. }
\end{gathered}
$$

According to jumping eq. (14), we have:

$$
\left(\sigma_{x}^{\infty}\right)_{\mathrm{I}}=\frac{(1+\alpha)}{(1-\alpha)}\left(\sigma_{x}^{\infty}\right)_{\mathrm{II}}+\frac{2 \sigma_{y}^{\infty}}{(1-\alpha)}(2 \beta-\alpha) .
$$

This means that there are only two independent load parameters $\left(\sigma_{x}^{\infty}\right)_{\text {II }}$ and $\sigma_{y}^{\infty}$. Due to the load parameter, $\sigma_{y}^{\infty}$ only produces uniform stress fields and has no effect on the stress $\sigma_{x}$ in material II if the load parameter $\left(\sigma_{x}^{\infty}\right)_{\text {II }}$ keeps constant. Hence, without losing generality, we only study the problem of $\sigma_{y}^{\infty}=0$. The second solution is that for a crack perpendicular to the interface with the uniform traction prescribed on the crack faces and all stress components vanish at infinity. Thus, we have:

$$
\begin{gathered}
\sigma_{x}+i \tau_{x y}=\Phi(z)+\overline{2 \Phi(z)}-\Omega(\bar{z})-(z-\bar{z}) \overline{\Phi^{\prime}(z)}=-\sigma, \text { at } z= \pm 0+i y,-a<y \longleftarrow b, \\
\sigma_{i j} \longrightarrow 0, \text { at infinity, }
\end{gathered}
$$

where $\sigma=\left(\sigma_{x}^{\infty}\right)_{\mathrm{II}}$. 
Using the function $I\left(z^{*}\right)$ and eq. (11), for symmetrical problem $\left(b_{y}=0\right)$, one obtains:

$$
\begin{gathered}
\Phi_{0}(z)=\frac{\mu_{2}}{\left(\kappa_{2}+1\right)} I(i z), \\
\Omega_{0}(z)=\frac{\mu_{2}}{\left(\kappa_{2}+1\right)} I(-i z)-\frac{2 \mu_{2}}{\left(\kappa_{2}+1\right)} i z I^{\prime}(-i z) .
\end{gathered}
$$

Substituting eqs (3a)-(c), (17) and (18) into eq. (16a), one obtains the following governing equation:

$$
\begin{gathered}
\left.\frac{\mu_{2}}{\kappa_{2}+1} 2 \overline{I^{+}(t)}+2 t \overline{I^{+}(t)}-I^{-}(t)\right]+I^{+}(t)-I^{-}(t)+\left(3 \Lambda_{2}-\Lambda_{1}\right) I(-t) \\
-12 \Lambda_{2} t I(-t)+4 t^{2} \Lambda_{2} I^{\prime \prime}(-t)=-\sigma \text { at } b<t<a .
\end{gathered}
$$

The governing eq. (19) is a function equation, which contains a set of unknown coefficients $\alpha_{m}$. The infinite series in eqs (9) and (11) can be approximated with a sufficient degree of accuracy by the corresponding truncated series. The crack surface is discretized into $M+1$ elements. The nodal points are given by the following expression:

$$
t_{k}=c+a_{0}\left(1+\cos \theta_{k}\right), \theta_{k}=\frac{k \pi}{(M+1)}, k=1,2, \ldots, M .
$$

According to the boundary collocation method, the governing eq. (19) should be satisfied on these nodal points. Then the governing equation is transformed into a set of linear algebraic equations. From these equations, one can get a set of unknown coefficients $\alpha_{m}(m=1$, $2, \ldots, M)$.

\subsection{Stress intensity factor}

The stress distribution ahead of the crack tip $B$ can be expressed as follows:

$$
\left.\sigma_{x}+i \tau_{x y}=\frac{\mu_{2}}{\kappa_{2}+1}\left[2 \overline{I(t)}+2 t \overline{\left(I^{\prime}(t)\right.}-I^{\prime}(t)\right)+\left(3 \Lambda_{2}-\Lambda_{1}\right) I(-t)-12 \Lambda_{2} t I^{\prime}(-t)+4 t^{2} \Lambda_{2} I^{\prime \prime}(-t)\right] .
$$

For the symmetric problem, above equation can be reduced to:

$$
\sigma_{x}=\frac{\mu_{2}}{\kappa_{2}+1}\left[2 I(t)+\left(3 \Lambda_{2}-\Lambda_{1}\right) I(-t)-12 \Lambda_{1} t I^{\prime}(-t)+4 t^{2} \Lambda_{2} I^{\prime \prime}(-t)\right]
$$

Now we have

$$
\begin{gathered}
K_{I}(B)=\lim _{r \rightarrow 0} \sqrt{2 \pi r} \sigma_{x}=-\frac{2 \mu_{2}}{\left(\kappa_{2}+1\right)} \sqrt{\pi a_{0}} \sum_{m=1}^{\infty}(-1)^{m} \alpha_{m}, \\
K_{\mathrm{I}}(A)=-\frac{2 \mu_{2}}{\left(\kappa_{2}+1\right)} \sqrt{\pi a_{0}} \sum_{m=1}^{\infty} \alpha_{m} .
\end{gathered}
$$

\subsection{T stress}

The stress fields ahead of the crack tip in an isotropic homogeneous elastic solid can be expressed as an asymptotic series:

$$
\sigma_{i j}=\frac{K_{\mathrm{I}}}{\sqrt{2 \pi r}} f_{i j}(\theta)+T \delta_{1 i} \delta_{1 j}
$$

The parameter $T$ defines the $T$ stress, which characterizes the second term of the Williams series. Linear elastic fracture mechanics is usually based on the assumption that the stress fields near the crack tip are dominated by $K$ field. However, much work has shown that a single parameter $K_{I}$ might not suffice to characterize the crack tip fields. Three point cracked bending specimen 
may produce a high triaxiality stress state, while the center cracked panel may produce low triaxiality stress state near the crack tip. The $T$ stress has significant effect on the triaxiality of the crack tip stress state. The compressive $T$ stress is related to a low triaxiality stress state. Hence, the $T$ stress plays an important role in the analysis of fracture process.

Near crack tip $B$, we have:

$$
\begin{gathered}
t=b-r, \xi=-\left(1+\frac{r}{a_{0}}\right) \\
I(t)=-\frac{1}{\sqrt{|\xi|^{2}-1}} \sum_{m=1}^{\infty} \alpha_{m}\left[\xi^{m}+\xi^{m-1} \sqrt{|\xi|^{2}-1}+\cdots\right]=-1 \sqrt{|\xi|^{2}-1} \sum_{m=1}^{\infty} \\
\alpha_{m} \xi^{m}-\sum_{m=1}^{\infty} \alpha_{m m} \xi^{m-1}+o\left(\sqrt{|\xi|^{2}-1}\right) .
\end{gathered}
$$

Substituting above equation into eq. (22) and letting $r$ approach zero, we obtain:

$$
T_{x}^{*}=\frac{\mu_{2}}{\kappa_{2}+1}\left\{2 \sum_{m=1}^{\infty}(-1)^{m} m \alpha_{m}+\left(3 \Lambda_{2}-\Lambda_{1}\right) I(-b)-12 \Lambda_{2} b I^{\prime}(-b)+4 b^{2} \Lambda_{2} I^{\prime \prime}(-b)\right\} .
$$

where $T_{x}^{*}$ is the $x$-component of $T$ stress contributed by the second solution of the symmetric problem. On the other hand, we have:

$$
\sigma_{x}+\sigma_{y}=4 \operatorname{Re}\{\Phi(z)\}=\frac{4 \mu_{2}}{\kappa_{2}+1}\left\{I(t)+\Lambda_{2}\left[I(t)-2 t I^{\prime}(t)\right]\right\} .
$$

A similar analysis leads to:

$$
T_{x}^{*}+T_{y}^{*}=\frac{4 \mu_{2}}{\kappa_{2}+1}\left\{\sum_{m=1}^{\infty}(-1)^{m} m \alpha_{m}+\Lambda_{2}\left[I(-b)-2 b I^{\prime}(-b)\right]\right\} .
$$

From the above equation and eq. (27), it follows:

$$
T_{y}^{*}=\frac{\mu_{2}}{\kappa_{2}+1}\left\{2 \sum_{m=1}^{\infty}(-1)^{m} m \alpha_{m}+\left(\Lambda_{2}+\Lambda_{1}\right) I(-b)+4 \Lambda_{2} b I^{\prime}(-b)-4 b^{2} \Lambda_{2} I^{\prime}(-b)\right\} .
$$

As we know, the second term of the Williams series in an isotropic homogeneous elastic solid only produces one stress component, which is parallel to the crack surface. Hence, we have:

$$
\sigma+\frac{\mu_{2}}{\kappa_{2}+1}\left[2 \sum_{m=1}^{\infty}(-1)^{m} m \alpha_{m}+\left(3 \Lambda_{2}-\Lambda_{1}\right) I(-b)-12 \Lambda_{2} b I^{\prime}(-b)+4 b^{2} \Lambda_{2} l^{\prime \prime}(-b)\right]=0 .
$$

Our calculation shows that eq. (31) is satisfied with very high accuracy $\left(\approx 10^{-12} \sigma-10^{-14} \sigma\right)$.

The total $T$ stress in the $y$ direction is described as:

$$
\begin{gathered}
T=\sigma_{y}^{\infty}+T_{y}^{*}=\sigma_{y}^{\infty}+q_{T}\left(\sigma_{x}^{\infty}\right)_{\mathrm{Il}} \\
q_{T}=\frac{\mu_{2}}{\kappa_{2}+1}\left\{2 \sum_{m=1}^{\infty}(-1)^{m} m \alpha_{m}+\left(\Lambda_{2}+\Lambda_{1}\right) I(-b)+4 \Lambda_{2} b I^{\prime}(-b)-4 b^{2} \Lambda_{2} I^{\prime \prime}(-b)\right\} /\left(\sigma_{x}^{\infty}\right)_{\mathrm{II}}
\end{gathered}
$$

The parameter $q_{T}$ characterizes the effect of the remote stress $\left(\sigma_{x}^{\infty}\right)_{\mathrm{II}}$ on the $T$ stress.

\section{CALCULATION RESULTS}

The calculations were carried out for different material pairs. The convergence of the series in eqs (9) and (11) was very quickly obtained. A typical example for material pairs aluminum-epoxy in the case of $b / a_{0}=1.0$ was tested. $M=60,90,120,150$ and 180 gave the same results of stress intensity factors at the crack tips $A$ and $B$ with six digits of accuracy and the coefficients 
Table 1. Stress intensity factors for a half plane

\begin{tabular}{ccccc}
\hline$b / a_{0}$ & $\begin{array}{c}K_{\mathrm{I}}(A) / \sigma \sqrt{\pi a_{0}} \\
\text { Present reults }\end{array}$ & $\begin{array}{c}K_{\mathrm{I}}(A) / \sigma \sqrt{\pi a_{0}} \\
\text { Cook and Erdogan[2] }\end{array}$ & $\begin{array}{c}K_{\mathrm{I}}(B) / \sigma \sqrt{\pi a_{0}} \\
\text { Present results }\end{array}$ & $\begin{array}{c}K_{\mathrm{I}}(B) / \sigma \sqrt{\pi a_{0}} \\
\text { Cook and Erdogan[2] }\end{array}$ \\
\hline 0.10 & 1.211 & 1.211 & 1.759 & 1.759 \\
0.15 & 1.183 & 1.183 & 1.575 & 1.575 \\
0.20 & 1.163 & 1.163 & 1.464 & 1.464 \\
0.50 & 1.097 & 1.097 & 1.204 & 1.204 \\
1.00 & 1.054 & 1.054 & 1.091 & 1.091 \\
4.00 & 1.009 & 1.009 & 1.011 & 1.011 \\
9.00 & 1.002 & 1.002 & 1.003 & 1.003 \\
\hline
\end{tabular}

$\alpha_{m}$ approaches to zero rapidly, as $m$ increases. For example, $\alpha_{1}=0.475, \alpha_{10}=-0.4909 \times 10^{-7}$, $\alpha_{100}=0.8964 \times 10^{-16}, \alpha_{180}=-0.1626 \times 10^{-16}$.

We have also checked eq. (19). We found that eq. (19) satisfied not only on the nodal points, but also on all of the middle points of the neighboured nodal points with 14 digits of accuracy. All of results given in this paper were calculated with $M=180$.

\subsection{Stress intensity factor}

The calculated stress intensity factors for a half plane are given in Table 1. For comparison, the results by Cook and Erdogan [2] are also listed in this table. The present results agree very well with their results. The calculated stress intensity factors for the material pairs aluminumepoxy in the plane strain are shown in Table 2 . The elastic constants are $\mu_{1} / \mu_{2}=23.08, \nu_{1}=0.3$, $\nu_{2}=0.35$. The present results completely agree with the results by Cook and Erdogan[2].

The stress intensity factors at the crack tip $B$ vs. the geometry parameter $\rho=b / a_{0}$ are plotted on Fig. 2 for the material pairs aluminum-epoxy. The nondimensional stress intensity factor $\bar{K}_{\mathrm{I}}(B)=K_{\mathrm{I}} / \sigma \sqrt{\pi a_{0}}$. When the crack in the weak, epoxy approaches the interface and the stress intensity factor at crack tip $B$ will approach zero due to the strong block from the stiff material aluminum.

It is worth noting that the stress intensity factor $\bar{K}_{\mathrm{I}}(B)$ at crack tip $B$ varies approximately according to a power law relationship. The following fitting equation is proposed:

$$
\begin{gathered}
\bar{K}_{\mathrm{I}}(B)=q\left(\frac{b}{a_{0}}\right)^{\gamma}, \\
\gamma=\left(\frac{1}{2}-\lambda\right)+\frac{1}{2}\left(\frac{1}{2}-\lambda\right)^{2},
\end{gathered}
$$

where parameter $\lambda$ is the lowest real eigenvalue in the range of $0<\lambda<1$ of the following characteristic equation

$$
\cos (\pi \lambda)+\frac{2(\alpha-\beta)}{1+\beta}(1-\lambda)^{2}-\frac{\alpha+\beta^{2}}{1-\beta^{2}}=0,
$$

where $\alpha$ and $\beta$ are Dundurs' bimaterial parameter. The parameter $\lambda$ defines the strength of the stress singularity ahead of the tip of a crack, which is perpendicular to and terminating at the bimaterial interface. For the material pairs aluminum-epoxy with a crack in the epoxy, one obtains $\lambda=0.33811, \alpha=0.91084$ and $\beta=0.20775$ in the plane strain condition.

Table 2. Stress intensity factors for an aluminium-epoxy bimaterial

\begin{tabular}{ccccc}
\hline$b / a_{0}$ & $\begin{array}{c}K_{\mathrm{I}}(A) / \sigma \sqrt{\pi a_{0}} \\
\text { Present results }\end{array}$ & $\begin{array}{c}K_{\mathrm{I}}(A) / \sigma \sqrt{\pi a_{0}} \\
\text { Cook and Erdogan[2] }\end{array}$ & $\begin{array}{c}K_{\mathrm{I}}(B) / \sigma \sqrt{\pi a_{0}} \\
\text { Present results }\end{array}$ & $\begin{array}{c}K_{\mathrm{I}}(B) / \sigma \sqrt{\pi a_{0}} \\
\text { Cook and Erdogan[2] }\end{array}$ \\
\hline 0.10 & 0.8985 & 0.8985 & 0.6674 & 0.6674 \\
0.15 & 0.9051 & 0.9051 & 0.7179 & 0.7179 \\
0.25 & 0.9165 & 0.9165 & 0.7838 & 0.7838 \\
1.00 & 0.9616 & 0.9616 & 0.9249 & 0.9249 \\
4.00 & 0.9929 & 0.9929 & 0.9912 & 0.9912 \\
9.00 & 0.9981 & 0.9981 & 0.9979 & 0.9979 \\
\hline
\end{tabular}




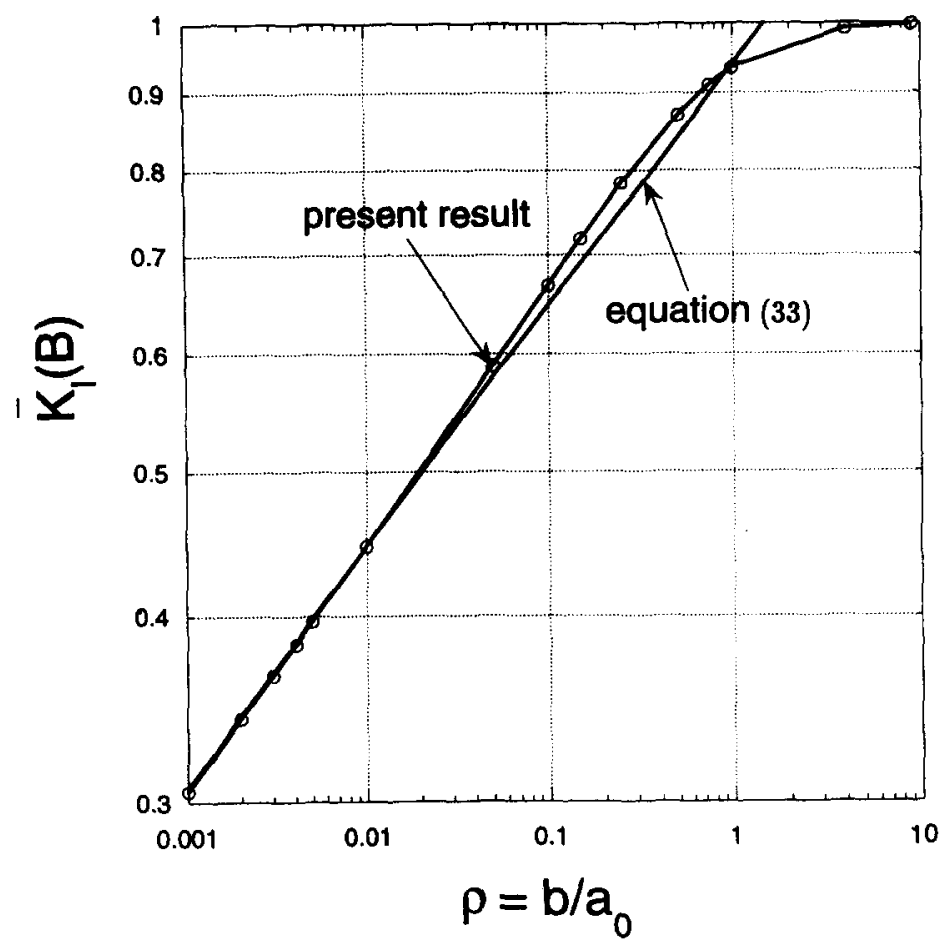

Fig. 2. Nondimensional stress intensity factor $\bar{K}_{\mathrm{I}}(B)=K_{\mathrm{I}}(B) / \sigma \sqrt{\pi a_{0}}$ vs the geometry parameter $\rho$ for material pairs aluminum-epoxy.

The curve given by eq. (33) is also plotted on Fig. 2. It is clear the fitting curve gives very good prediction when the parameter $\rho=b / a_{0}$ less than 0.05 . Figure 3 shows the nondimensional stress intensity factors vs the parameter $\rho=b / a_{0}$ for the material pairs epoxy-boron in plane strain. The materials constants are $\mu_{1} / \mu_{2}=0.007223, \nu_{1}=0.35, v_{2}=0.3$. For this case, $\lambda=0.9258, \alpha=-0.98456$ and $\beta=-0.22678$ in the plane strain condition. The crack is now within stiff material Boron. The stress intensity factor at the crack tip $B$ increases rapidly when

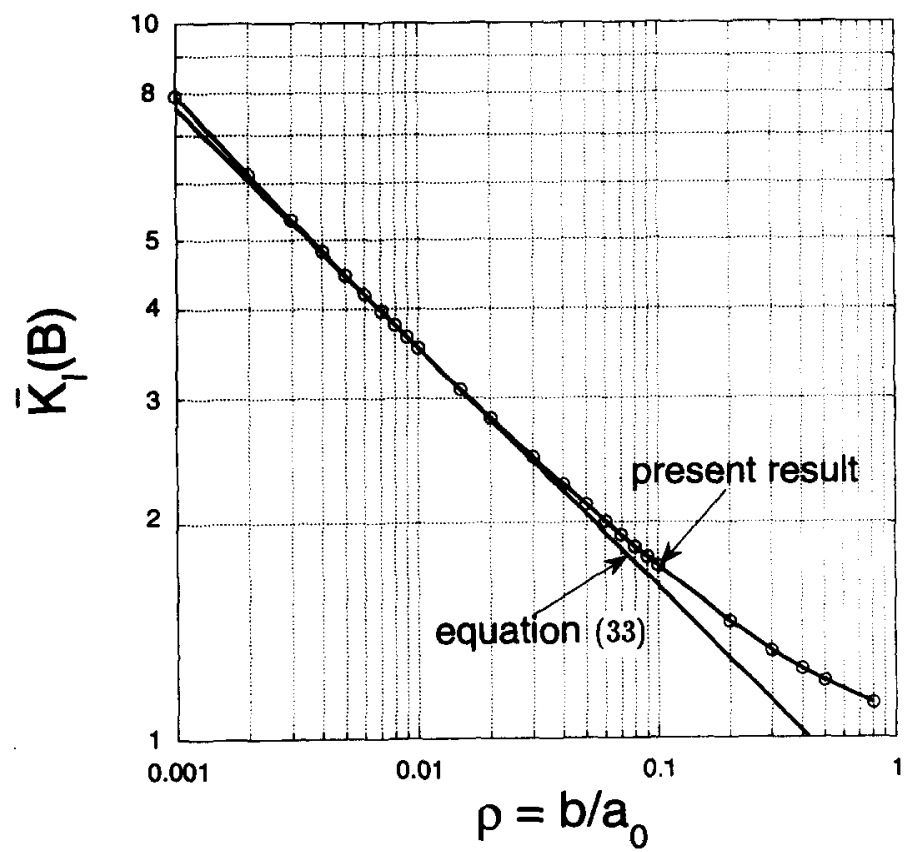

Fig. 3. The relationship between the nondimensional stress intensity factor $\bar{K}_{\mathrm{I}}(B)=K_{\mathrm{I}}(B) / \sigma \sqrt{\pi a_{0}}$ and the geometry parameter $\rho$ for material pairs epoxy-boron. 


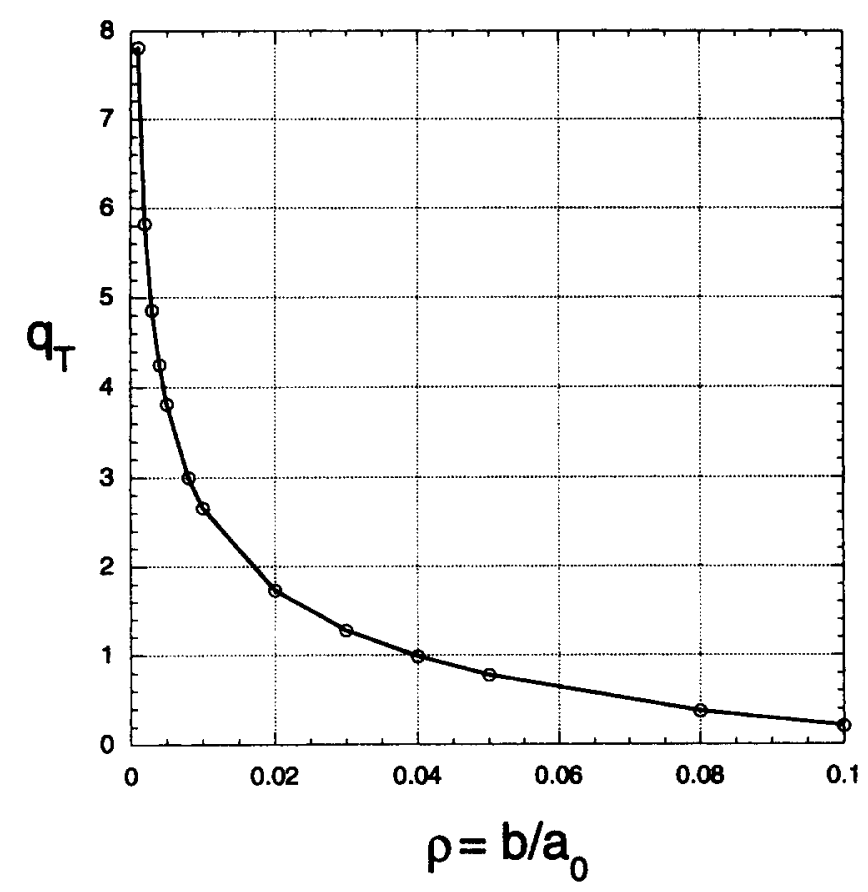

Fig. 4. Parameter $q_{T}$ variation for material pairs aluminum-epoxy.

the geometry parameter $\rho$ approaches to zero. The fitting curve by eq. (33) is in good agreement with the present results as $\rho \leq 0.1$.

\subsection{Parameter $q_{T}$}

The parameter $q_{T}$, which characterizes the effect of the remote stress $\left(\sigma_{x}^{\infty}\right)_{\mathrm{II}}$ upon the $T$ stress is plotted in Fig. 4 and 5 for material pairs aluminum-epoxy and epoxy-boron, respectively. For the material pairs aluminum-epoxy, the parameter $q_{T}$ is always positive and increases as the crack approaches the interface. On the other hand, for the material pairs epoxy-boron, this parameter is always negative and decreases as the crack approaches to the interface. This

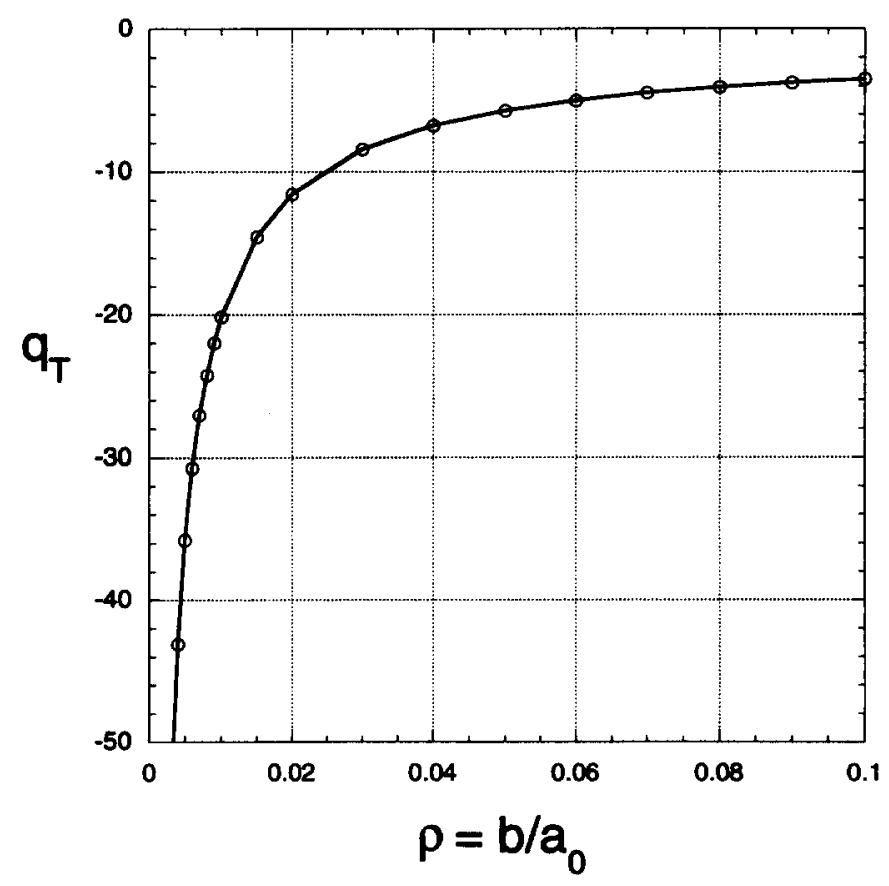

Fig. 5. Parameter $q_{T}$ variation for material pairs epoxy-boron. 


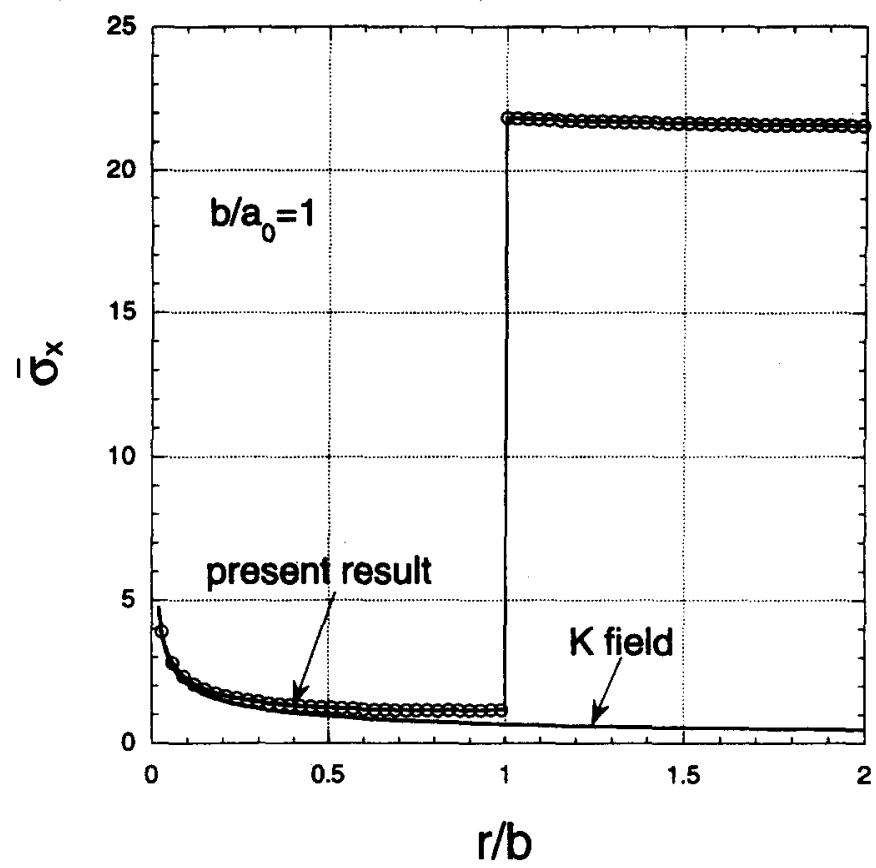

Fig. 6. Normal stress $\sigma_{x}$ distribution ahead of the crack tip $B$ for material pairs aluminum-epoxy in the case of $b / a_{0}=1$. Here $x=0, r$ is the distance from crack tip $B$ and $\bar{\sigma}_{x}=\sigma_{x} / \sigma$.

means that the effect of the remote stress $\left(\sigma_{x}^{\infty}\right)_{\text {II }}$ upon the $T$ stress becomes significant when the crack is growing towards to the interface.

\subsection{Stress distribution ahead of crack tip $B$}

Figure 6 and 7 show the stress distributions ahead of the crack tip $B$ for the material pairs aluminum-epoxy in the case of $b / a_{0}=1$. It is clear that the normal stress $\sigma_{x}$ is dominated by the $K$ field in the region of $0<r / b<0.1$, meanwhile the normal stress $\sigma_{y}$ is characterized by the $K$ field plus the $T$ stress in the same region. On the other hand, the normal stress $\sigma_{x}$ has very big-

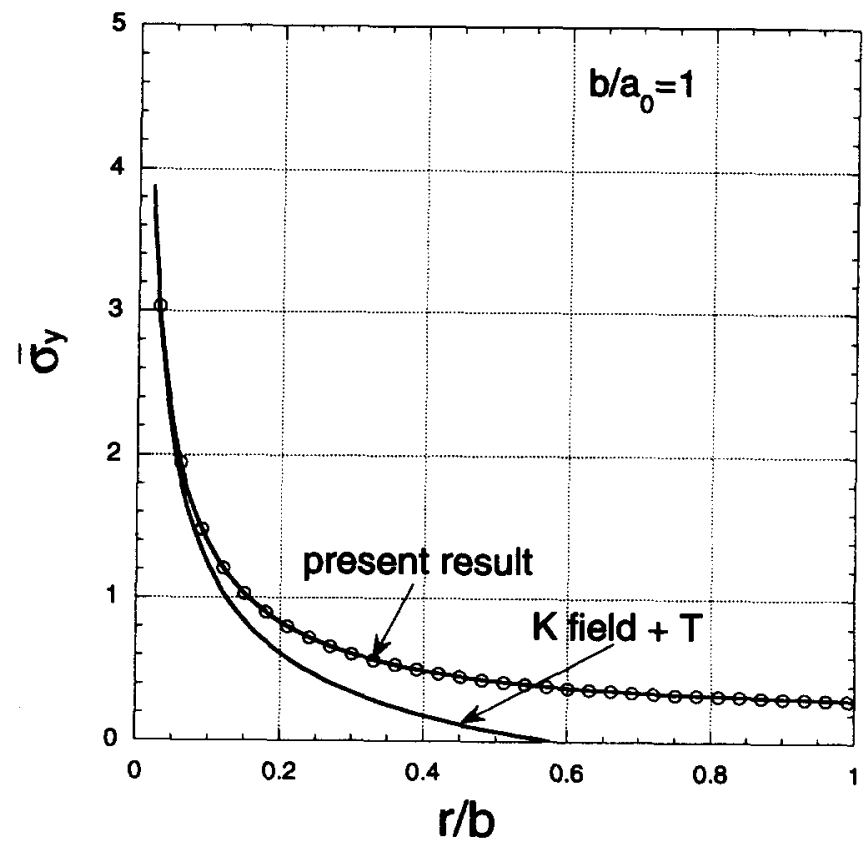

Fig. 7. Normal stress $\sigma_{y}$ distribution ahead of the crack tip $B$ for material pairs aluminum-epoxy in the case of $b / a_{0}=1$ and $\bar{\sigma}_{y}=\sigma_{y} / \sigma$. 


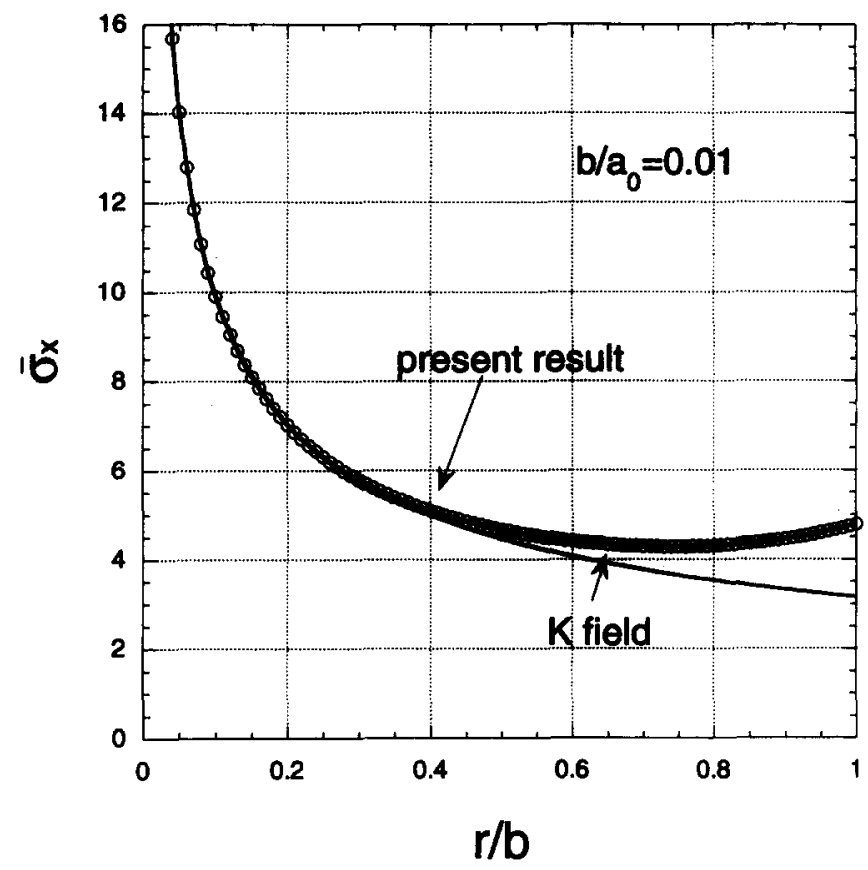

Fig. 8. Normal stress $\sigma_{x}$ distribution ahead of the crack tip $B$ for material pairs aluminum-epoxy in the case of $b / a_{0}=0.01$.

ger jump across the interface due to the bigger mismatch in the elastic constants of the bimaterial aluminum-epoxy. In the stiff material aluminum, the normal stress $\sigma_{x}$ at the interface is as large as 20 times the stress $\sigma_{x}$ in the weaker material epoxy.

Figures 8 and 9 show the stress distributions ahead of the crack tip $B$ for the case of $b /$ $a_{0}=0.01$. The result given by $K$ field agrees very well with the present result for normal stress $\sigma_{x}$ in the region of $0<r / b<0.4$. However, for the normal stress $\sigma_{y}$ the $K$ field is remarkably deviated with the present result. It is clearly revealed that the $T$ stress effect is very important

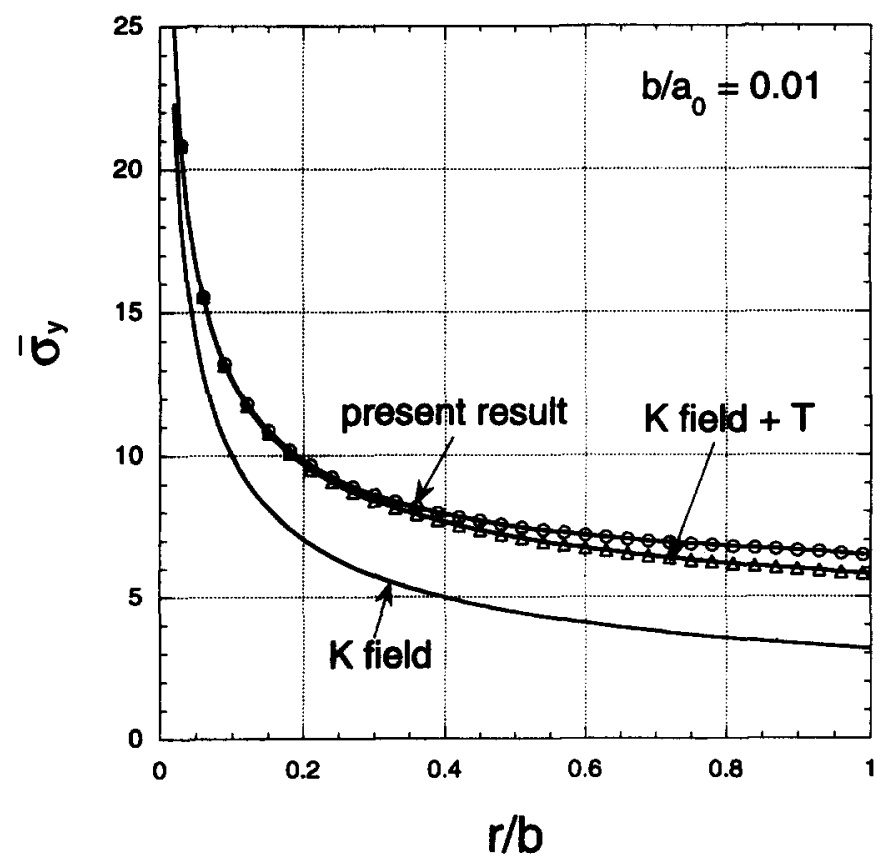

Fig. 9. Comparison of the normal stress $\sigma_{y}$ with the $K$ field and the $K$ field plus $T$ stress for material pairs aluminum-epoxy in the case of $b / a_{0}=0.01$. 


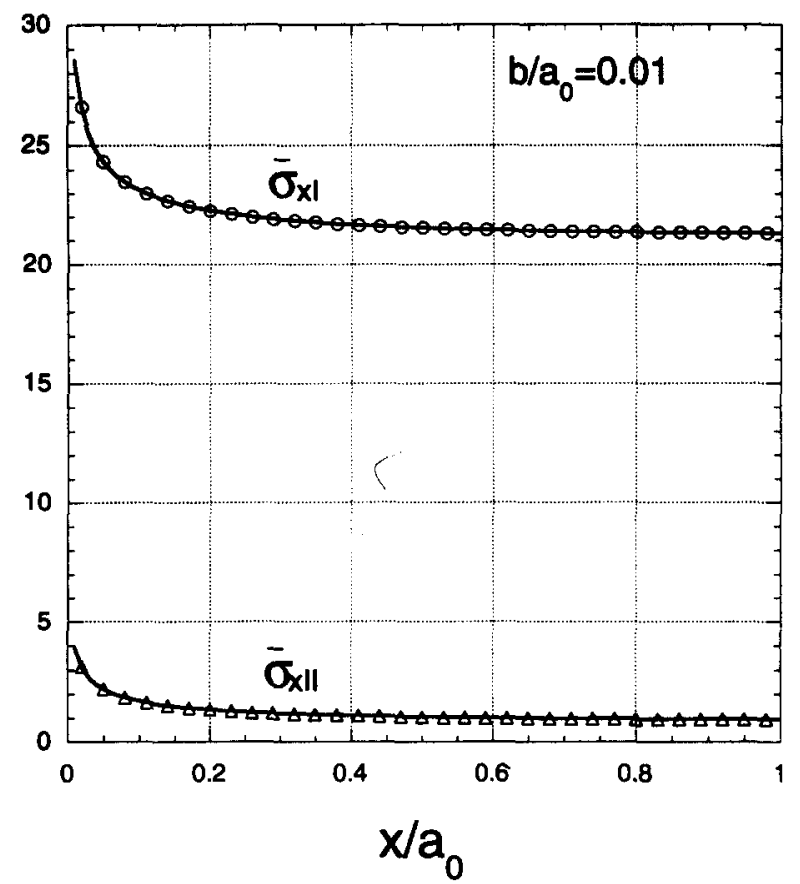

Fig. 10. Normal stress $\sigma_{x}$ distribution along the interface for material pairs aluminum-epoxy in the case of $b / a_{0}=0.01$.

for material pairs aluminum-epoxy. The stress distributions along the interface are plotted on Fig. 10.

Now we look at the situation when the crack is within the stiff material. Figures 11 and 12 show the stress fields ahead of the crack tip $B$ for the bimaterial epoxy-boron in the case of $b$ $a_{0}=0.1$. The normal stresses $\sigma_{x}$ and $\sigma_{y}$ are characterized by $K$ field and the $K$ field plus $T$ stress, respectively, in the region of $0<r / b<0.4$. The $K$ field cannot described the normal stress $\sigma_{y}$ as shown in Fig. 12.

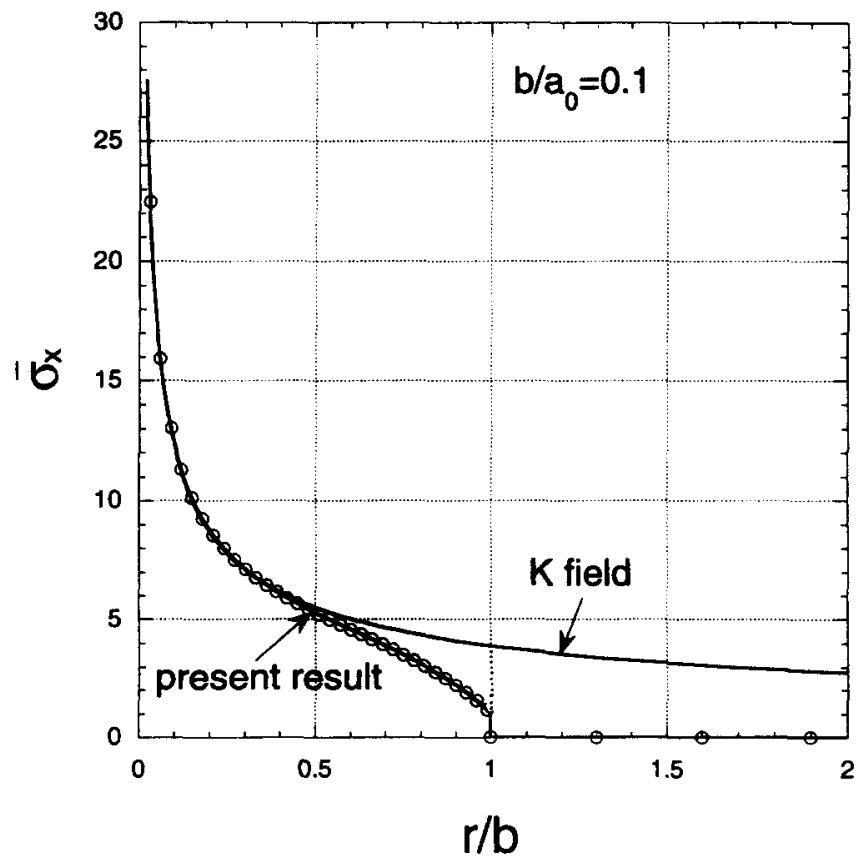

Fig. 11. Normal stress distribution $\sigma_{x}$ ahead of the crack tip $B$ for material pairs epoxy-boron in the case of $b / a_{0}=0.1$. 


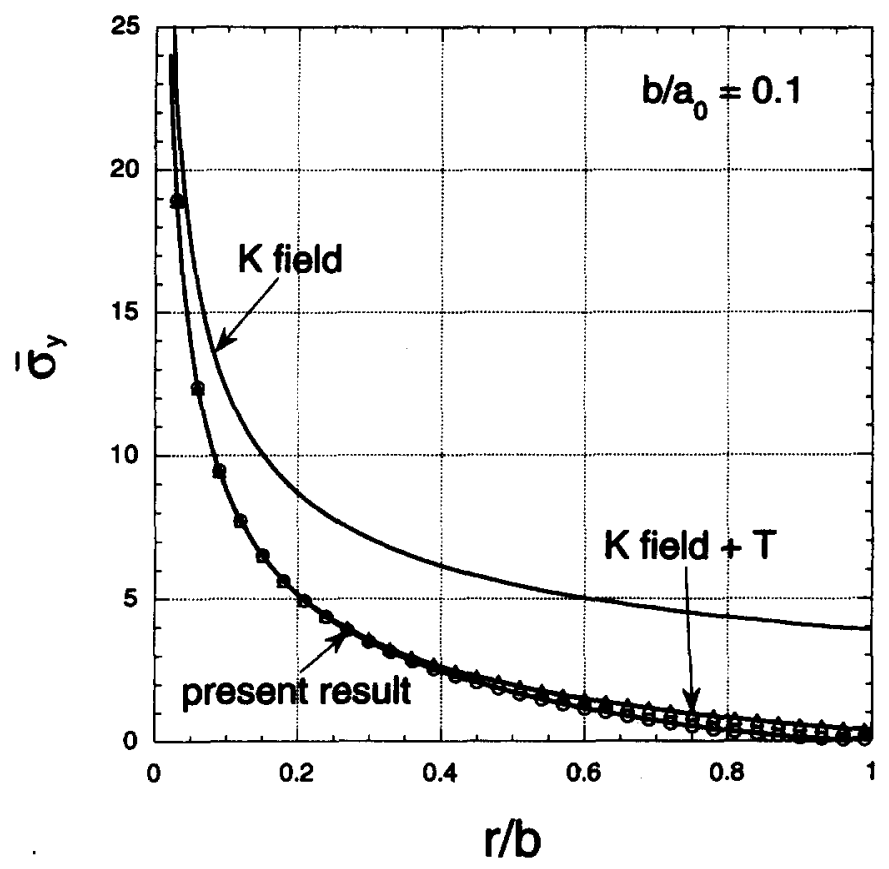

Fig. 12. Comparisons of the normal stress $\sigma_{y}$ with the $K$ field and the $K$ field plus $T$ stress for material pairs epoxy-boron in the case of $b / a_{0}=0.1$.

\section{CONCLUSION AND DISCUSSION}

From this study, we can draw following conclusions:

1. The problem of a crack perpendicular to an interface which is not intersected with the interface is accurately solved using the dislocation simulation approach in this paper.

2. The normal stress $\sigma_{x}$ ahead of the crack tip which is near the interface is characterized by the $K$ fields in the region of $0<r / b<0.4$ and the normal stress $\sigma_{y}$, which is parallel to the crack surface, is dominated by the $K$ field plus $T$ stress in the same region for $b / a_{0} \leq 0.1$.

3. As the crack approaches the interface, the parameter $b$ becomes an important length scale, which characterizes the dominated zone of the $K$ field and $K$ field plus the $T$ stress field.

4. The stress $\sigma_{x}$ has a jump across the interface due to the elastic constant mismatch. For materials pairs aluminum-epoxy, the stress jump can be as large as about 20 times of the remote stress $\left(\sigma_{x}^{\infty}\right)_{\mathrm{II}}$, which is the same order magnitude of the jump in the remote stress $\sigma_{x}^{\infty}$, $(1+\alpha) /(1-\alpha)$. For materials pairs epoxy-boron, the stress jump is only same order of the remote stress $\left(\sigma_{x}^{\infty}\right)_{\mathrm{II}}$, owing the parameter $\alpha=-0.98456$.

5. The $T$ stress near the crack tip can be determined by the remote stress $\sigma_{y}^{\infty}$ plus $q_{T}$ times the remote stress $\left(\sigma_{x}^{\infty}\right)_{\mathrm{II}}$, where the parameter $q_{T}$ characterizes the effect of the remote stress $\left(\sigma_{x}^{\infty}\right)_{\text {II }}$ on the $T$ stress. If the crack is in the weaker material, the parameter $q_{T}$ is always positive, otherwise the parameter is always negative as the crack is in the stiff material.

The above conclusions are based on the analysis for an infinite bimaterial, which is bonded by two half planes containing a finite crack perpendicular to the interface and subject to uniform remote load at infinity. For finite bimaterial and complex loading, the situation will be complicated. In order to gain a better understanding for fracture characteristics of a crack perpendicular to the interface further investigation is needed. 


\section{REFERENCES}

1. Zak, A. R. and Williams, M. L., Crack point singularities at a bimaterial interface. Journal of Applied Mechanics, $1963,30,142-143$.

2. Cook, T. S. and Erdogan, F., Stress in bonded material with a crack perpendicular to the interface. International Journal of Engineering Sciences, 1972, 10, 677-697.

3. Erdogan, F. and Biricikoglu, B., Two bonded half plane with a crack going through the interface. International Engineering Science, 1973, 11, 745-766.

4. Bogy, D. B., On the plane elastostatic problem of a loaded crack terminating a material interface. Journal of International Fracture, 1971, 38, 911-918.

5. Wang, W. C. and Chen, J. T., Theoretical and experimental reexamination of a crack perpendicular to and terminating at the bimaterial interface. Journal of Strain Analysis, 1993, 28, 53-61.

6. Lin, K. Y. and Mar, J. W., Finite element analysis of a stress intensity factors for crack at a bimaterial interface. International Journal of Fracture, 1976, 12, 521-531.

7. Ahmad, J., A micromechanics analysis of crack in unidirectional fiber composite. Journal of Applied Mechanics, $1991,58,964-972$.

8. Meguid, S. A., Tan, M. and Zhu, Z. H., Analysis of crack perpendicular to bimaterial interface using a novel finite element. International Journal of Fracture, 1995, 75, 1-25.

9. Chen, D. H., A crack normal to and terminating at a bimaterial interface. Engineering Fracture Mechanics, 1994, 49, 517-523.

10. Ståhle, P., Gunnars, J. and Delfin, P., Crack path in a weak elastic layer covering a beam. Acta Mechanica Solid Sinica, 1995, 8, 579-583.

11. Delfin, P., Gunnars, J. and Ståhle, P., Effect of elastic mismatch on the growth of a crack initially terminated at an interface in elastic plastic materials. Fatigue Fracture, Engineering Materials and Structures, 1995, 18, 1201-1212.

12. Dundurs, J., In Mathematics of Dislocation, ed. T. Mura. ASME, New York, 1969, pp. 70-115.

13. Tamate, O., International Journal of Fracture 1968, 4, 357.

14. Tamate, O. and Kurihara K., International Journal of Fracture 1970, 6, 410.

15. Suo, Z., Singularities interacting with interface and cracks. International Journal of Solids and Structures, 1989, 25, 1133-1142.

16. Gladwell, G. M. L., Contact Problem in the Classical Theory of Elasticity. Sijthoff and Noordhoff, Gronongen, 1980 , pp. 61-64. 